\title{
MOOCs y estudios de género. Mujeres coraje a modo de ejemplo
}

\section{Juncal Caballero Guiral ${ }^{\mathrm{a}}$ y Carmen María Fernández Nadal ${ }^{\mathrm{b}}$}

${ }^{a}$ Departamento de Historia, Geografía y Arte, Instituto Universitario de Estudios Feministas y de Género Purificación Escribano, Universitat Jaume I, mguiral@uji.es, ${ }^{b}$ Departamento de Historia, Geografía y Arte, Instituto Universitario de Estudios Feministas y de Género Purificación Escribano, Universitat Jaume I, nadal@,uji.es.

\begin{abstract}
This paper aims to analyze the impact of Gender Studies in MOOC Courses. For three years, at the University Jaume I in Castellon, and with the support of CENT, we have developed three online, massive and open online courses on Gender Studies, entitled "Courageous Women". This has allowed us to disseminate Feminist and Gender Studies with a new learning tool, and, at the same time, we have been able to reach a wide range of people interested in these studies. The topic and the working language influence, completely, a student body that is mainly female and Spanish speaking. All in all, this analysis is the result of an experience developed along three years, which has allowed us to prove the possibilities that this new methodology can offer, but, also, the problems we have to deal with.
\end{abstract}

Keywords: MOOC, Gender Studies, Learning, Methodology, Transversal Approach.

\begin{abstract}
Resumen
El presente trabajo tiene como objetivo analizar la repercusión que poseen los estudios de género en los cursos MOOC. Durante tres años, desde la Universitat Jaume I de Castellón, y con la ayuda del CENT, se han ido realizando tres ediciones de cursos online, masivos y abiertos versados en los estudios de género bajo el título "Mujeres coraje". Lo que nos ha permitido difundir los estudios feministas y de género con una nueva herramienta educativa y, a su vez, acceder a un amplio abanico de personas interesadas en dichos estudios. La materia tratada y la lengua vehicular incidirán, plenamente, en un estudiantado mayoritariamente femenino e hispanoblante. Este análisis es, en definitiva, el resultado de una experiencia desarrollada durante tres años y que nos ha llevado a comprobar las posibilidades que esta nueva metodología ofrece pero, también, de las dificultades a las que debemos enfrentarnos.
\end{abstract}

Palabras clave: MOOC, estudios de género, educación, metodología, transversalidad. 


\section{Introducción}

Los Cursos Online Abiertos Masivos (MOOC/COMA) han posibilitado desde el año 2012 una vía innovadora para el aprendizaje educativo a través de un acceso gratuito y sin límites. La andadura de los MOOCs, como ya se ha comentado ampliamente por la comunidad científica, es relativamente reciente (Luján, 2012). Esta herramienta educativa se ha convertido en una ventana a nuevos escenarios formativos, de esta forma se han creado plataformas exclusivas para albergarlos. Una parte son cobijadas por las propias universidades y, en otros casos, han nacido amparadas por sectores económicos privados, como puede ser el caso del proyecto de formación en línea en el ámbito iberoamericano, Miríada X.

La Universitat Jaume I de Castellón, como otros centros de educación superior, puso en marcha en 2013 una convocatoria de este tipo de cursos y habilitó un espacio propio gracias al impulso realizado por el Centre d'Educació i Noves Tecnologies (CENT). El Instituto Universitario de Estudios Feministas y de Género Purificación Escribano, de la misma universidad, consideró fundamental apostar por este tipo de herramientas para difundir los estudios feministas. Por ello, tras un debate interno, se propuso desde el instituto indagar en las diferentes posibilidades y comprobar el peso que este tipo de estudios tenía entonces en las plataformas existentes. De esta manera se pudo detectar una falta de contenidos de género en abierto y online. Somos conscientes, tres años después, de que el panorama ha cambiado, afortunadamente, aun así el goteo de cursos bajo este tipo de temáticas es menos fluido que en otras áreas de conocimiento.

El diseño y ejecución de este tipo de cursos en el medio educativo actual ha despertado grandes expectativas, aprovechadas por muchos y muchas, aunque es solo accesible a una parte del cosmos interconectado, aquella con acceso a Internet (Downes, 2012). De igual modo, no podemos obviar los problemas de sostenibilidad que generan desde el principio. Las nuevas tecnologías nos están dado la oportunidad de compartir y generar en tiempo récord, pero en muchas ocasiones, como esta, es necesaria la implicación docente de manera desinteresada. Creer en el proyecto y, sobre todo, creer que es posible. Resulta fundamental para desarrollar plenamente este tipo de innovaciones, más teniendo en cuenta los problemas de financiación que genera este tipo de iniciativas cuya filosofía se basa en el aprendizaje gratuito, masivo y abierto y las implicaciones técnicas que ello conlleva para quienes se embarcan en este tipo de aventuras educativas.

\section{Objetivos}

El objetivo principal de este trabajo es indagar en el potencial de los Cursos Online Abiertos Masivos (MOOC/COMA) dentro del ámbito de la docencia y la investigación en estudios feministas y de género. Y, por supuesto, evaluar la propuesta educativa que a lo largo de tres cursos académicos se ha puesto en marcha.

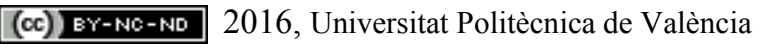




\section{Desarrollo de la innovación}

En los últimos años se han llevado a cabo diferentes experiencias bajo el epígrafe «Mujeres coraje». Estos cursos se han diseñado como pequeños lugares en los que un gran número de usuarios y usuarias interrelacionan compartiendo conocimientos. Teniendo en cuenta que podemos identificar dos tipos diferentes de MOOC «cMOOC, de raíz conectivista y que empezaron en el 2008 de la mano de Cormier, Siemens y Downes. xMOOC, desarrollados por universidades en Estados Unidos y actualmente en expasión. Estos cursos están basados en modelos tradicionales de aprendizaje mediante lecciones (grabadas), test de autoevaluación y completando pequeñas tareas...» (Atenas, 2015: 4-5).

La idea inicial era generar un cMOOC y huir del clásico xMOOC, limitado a pruebas de corrección objetiva, ya que nuestro fin era construir conocimiento de una manera colaborativa formando redes que debatieran sobre este tema transversal y fundamental. Pero las dificultades de llevar a término este planteamiento nos han situado en un camino intermedio que a continuación detallaremos.

\subsection{Mujeres coraje. Un diseño innovador para los estudios de género}

El planteamiento inicial partió de una idea sencilla pero, a la vez, lo suficientemente atractiva como para llegar a un amplio número de personas interesadas en este tipo de estudios, sin que fuera necesario unos requisitos mínimos para acceder al curso. Para ello, nos alejamos de un constructo teórico para fomentar el análisis a partir de ejemplos concretos. Consideramos necesario visibilizar a figuras públicas y anónimas en ámbitos diferentes pero con una característica en común: el haber sido capaces de desafiar a la historia patriarcal.

Es este el punto de partida que concretamos en un primer curso titulado «Mujeres coraje. Desafíos en la historia». En él se abordaba desde los ámbitos históricos, literarios, artísticos y filosóficos a figuras como Antígona, Josefa Bosch «La Pardala», Charlotte Perkins Gilman, Federica Monstseny, Rosario Castellanos o Regina Galindo.

Los dos siguientes cursos, bajo el amparo del epígrafe «Mujeres coraje» quisieron incidir, aun siguiendo una línea similar, en temas como la transgresión y los estereotipos. Dos aspectos que, a nuestro entender, son fundamentales en la interpretación del papel que las mujeres han jugado tradicionalmente.

El segundo curso titulado «Mujeres coraje. Maestras de la transgresión» abordó el análisis de las diferentes figuras teniendo en cuenta cómo fueron capaces de transgredir las normas imperantes. Estas figuras fueron las Mujeres mágicas, la Mujer vampírica, Maya Deren, Pauline Réage, Louise Bourgeois o el Grupo Femen.

El tercer y último curso, hasta la fecha, se titula «Mujeres coraje. Subvirtiendo estereotipos». Pensar en ellas es introducirse en un mundo repleto de estereotipos que han ido, poco a poco, configurando un ideal, tanto positivo como negativo, de «la mujer». Pero

2016, Universitat Politècnica de València

Congreso In-Red (2016) 
también al pensar en ellas nos encontramos con un gran número que con su osadía y fuerza supieron subvertir los estereotipos. En este curso se ha introducido, tras un cambio metodológico, un pequeño vídeo explicativo sobre qué son y cómo se subvierten los mismos para, de esta manera, acercarnos a figuras como Paul B. Preciado, las Fajas negras, Alison Lapper, Alice James o Mariana Grajales.

Todas las figuras tratadas se insertan en la rama de las Artes y Humanidades, analizando de manera transversal el papel que las mujeres jugaron en la Historia -desafiándola, transgrediéndola o subvirtiéndola. De esta manera, se proporcionaba a los y las interesadas las herramientas básicas para iniciarse en la investigación en estudios feministas y de género.

\subsection{Primera valoración del impacto de los MOOCs "Mujeres coraje"}

El número de matriculación de un MOOC, al tener en cuenta su carácter masivo, es superior a las cifras que habitualmente nos encontramos en la formación reglada. Por ello, el número alcanzado es superior a cursos tradicionales pero inferior a la media que tienen otros MOOCs de temáticas diferentes y ubicados en plataformas con mayor difusión. Debemos tener en cuenta no sólo estos aspectos sino también el hecho de que es un curso dirigido a un grupo específico de personas interesadas en estos temas.

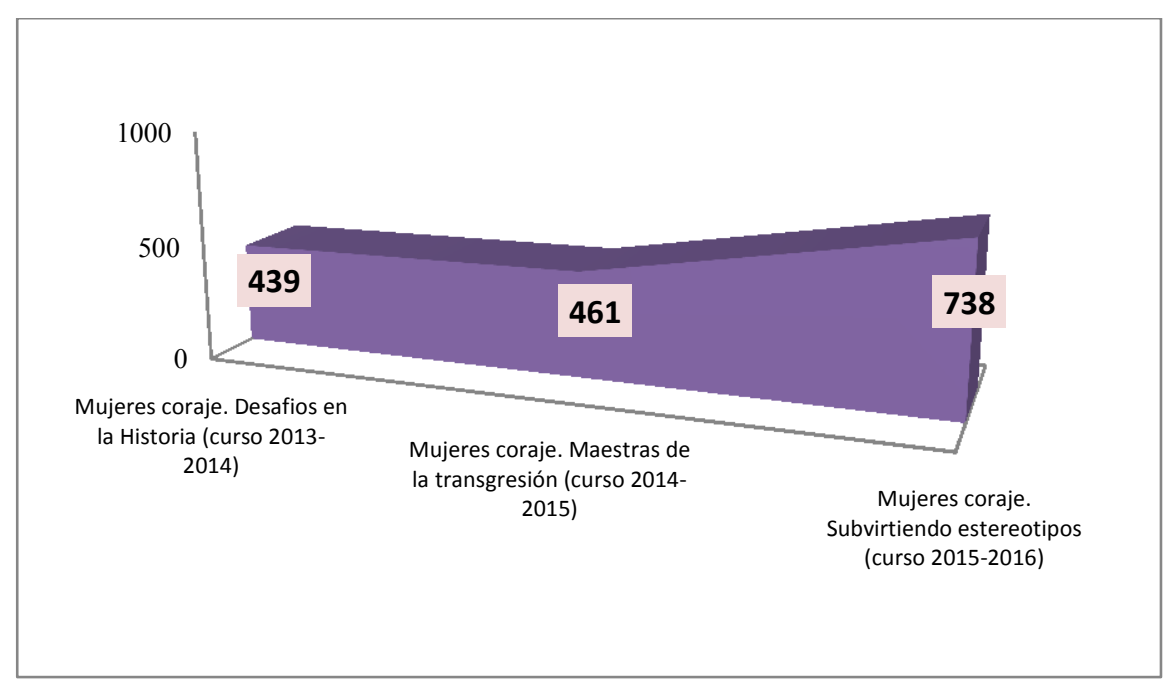

Fig. 1. Número de inscipciones en los diferentes cursos «Mujeres coraje»

Fuente: Elaboración propia con los datos facilitados por el CENT (UJI).

Al finalizar la segunda edición se detectó un estancamiento en la matrícula que nos alertó sobre el posible desinterés y la necesidad de realizar cambios metodológicos. Se trataba de adaptarnos a la formación que nuestro estudiantado demandaba. Teniendo en cuenta este

2016, Universitat Politècnica de València 
hecho y con las modificaciones efectuadas, ha sido gratificante comprobar cómo el número de matriculadas y matriculados ha aumentado considerablemente, superando las expectativas del grupo de dirección -como puede observarse en la Fig. 1. Si bien, no ha sido la difusión el motor que ha alimentado este incremento. Cabe reseñar que en este último año nos hemos encontrado con un $9 \%$ de estudiantado matriculado o graduado en nuestra propia universidad.

Asimismo, y como podemos observar tanto en la Tabla 1 como en la Fig. 2, mayoritariamente las personas inscritas tienen como lugar de residencia un país hispanohablante -España y América Latina. Al realizarse el curso con el español como lengua vehicular, ello conlleva una restricción y una limitación para aquellas personas que, si bien están interesadas en las cuestiones de género, no pueden seguir el curso convenientemente.

Tabla 1. Porcentaje de estudiantes por país de residencia

\begin{tabular}{|c|c|c|c|}
\hline & $\begin{array}{l}\text { Mujeres coraje. Desafíos en la } \\
\text { Historia (curso 2013-2014) }\end{array}$ & $\begin{array}{l}\text { Mujeres coraje. Maestras de la } \\
\text { transgresión (curso 2014-2015) }\end{array}$ & $\begin{array}{l}\text { Mujeres coraje. Subvirtiendo } \\
\text { estereotipos (curso 2015-2016) }\end{array}$ \\
\hline Alemania & $0,46 \%$ & $1,23 \%$ & $0,00 \%$ \\
\hline Argentina & $1,82 \%$ & $4,67 \%$ & $3,12 \%$ \\
\hline Austria & $0,23 \%$ & $0,00 \%$ & $0,00 \%$ \\
\hline Bélgica & $0,00 \%$ & $0,74 \%$ & $0,14 \%$ \\
\hline Bolivia & $0,23 \%$ & $0,25 \%$ & $0,27 \%$ \\
\hline Brasil & $0,46 \%$ & $0,25 \%$ & $0,14 \%$ \\
\hline Canadá & $0,46 \%$ & $0,00 \%$ & $0,14 \%$ \\
\hline Chile & $1,14 \%$ & $3,19 \%$ & $1,90 \%$ \\
\hline Chipre & $0,00 \%$ & $0,00 \%$ & $0,14 \%$ \\
\hline Colombia & $5,24 \%$ & $3,19 \%$ & $3,79 \%$ \\
\hline Costa Rica & $1,14 \%$ & $0,49 \%$ & $0,41 \%$ \\
\hline Croacia & $0,23 \%$ & $0,00 \%$ & $0,00 \%$ \\
\hline Dinamarca & $0,00 \%$ & $0,00 \%$ & $0,14 \%$ \\
\hline Ecuador & $0,23 \%$ & $0,49 \%$ & $0,27 \%$ \\
\hline Egipto & $0,00 \%$ & $0,25 \%$ & $0,00 \%$ \\
\hline El Salvador & $2,51 \%$ & $0,00 \%$ & $0,14 \%$ \\
\hline España & $71,53 \%$ & $72,48 \%$ & $77,10 \%$ \\
\hline EEUU & $0,68 \%$ & $0,74 \%$ & $0,54 \%$ \\
\hline Francia & $0,23 \%$ & $0,49 \%$ & $0,54 \%$ \\
\hline Grecia & $0,00 \%$ & $0,00 \%$ & $0,14 \%$ \\
\hline Guatemala & $0,23 \%$ & $0,00 \%$ & $0,14 \%$ \\
\hline Holanda & $0,23 \%$ & $0,00 \%$ & $0,00 \%$ \\
\hline Honduras & $0,00 \%$ & $0,00 \%$ & $0,27 \%$ \\
\hline Irlanda & $0,00 \%$ & $0,25 \%$ & $0,00 \%$ \\
\hline Japón & $0,00 \%$ & $0,25 \%$ & $0,00 \%$ \\
\hline México & $4,33 \%$ & $7,13 \%$ & $4,34 \%$ \\
\hline Nicaragua & $0,91 \%$ & $0,00 \%$ & $0,00 \%$ \\
\hline Noruega & $0,23 \%$ & $0,00 \%$ & $0,00 \%$ \\
\hline Paraguay & $0,23 \%$ & $0,00 \%$ & $0,14 \%$ \\
\hline Perú & $2,05 \%$ & $1,97 \%$ & $2,98 \%$ \\
\hline Polonia & $0,00 \%$ & $0,25 \%$ & $0,00 \%$ \\
\hline
\end{tabular}

(c) EY-NC-ND 2016, Universitat Politècnica de València

Congreso In-Red (2016) 
MOOCs y estudios de género. Mujeres coraje a modo de ejemplo

\begin{tabular}{|l|c|c|c|} 
Portugal & $0,46 \%$ & $0,25 \%$ & $0,00 \%$ \\
\hline Puerto Rico & $0,46 \%$ & $0,00 \%$ & $0,95 \%$ \\
\hline Reino Unido & $0,68 \%$ & $0,49 \%$ & $0,81 \%$ \\
\hline Rep. Checa & $0,23 \%$ & $0,00 \%$ & $0,00 \%$ \\
\hline Rep. Dominicana & $0,68 \%$ & $0,25 \%$ & $0,00 \%$ \\
\hline Rumanía & $0,46 \%$ & $0,00 \%$ & $0,00 \%$ \\
\hline Rusia & $0,00 \%$ & $0,25 \%$ & $0,00 \%$ \\
\hline Serbia & $0,00 \%$ & $0,25 \%$ & $0,00 \%$ \\
\hline Suecia & $0,46 \%$ & $0,00 \%$ & $0,00 \%$ \\
\hline Uruguay & $0,91 \%$ & $0,25 \%$ & $0,95 \%$ \\
\hline Venezuela & $0,91 \%$ & $0,00 \%$ & $0,27 \%$ \\
\hline
\end{tabular}

facilitados por el CENT (UJI).

(cc) EY-NC-ND 2016, Universitat Politècnica de València 


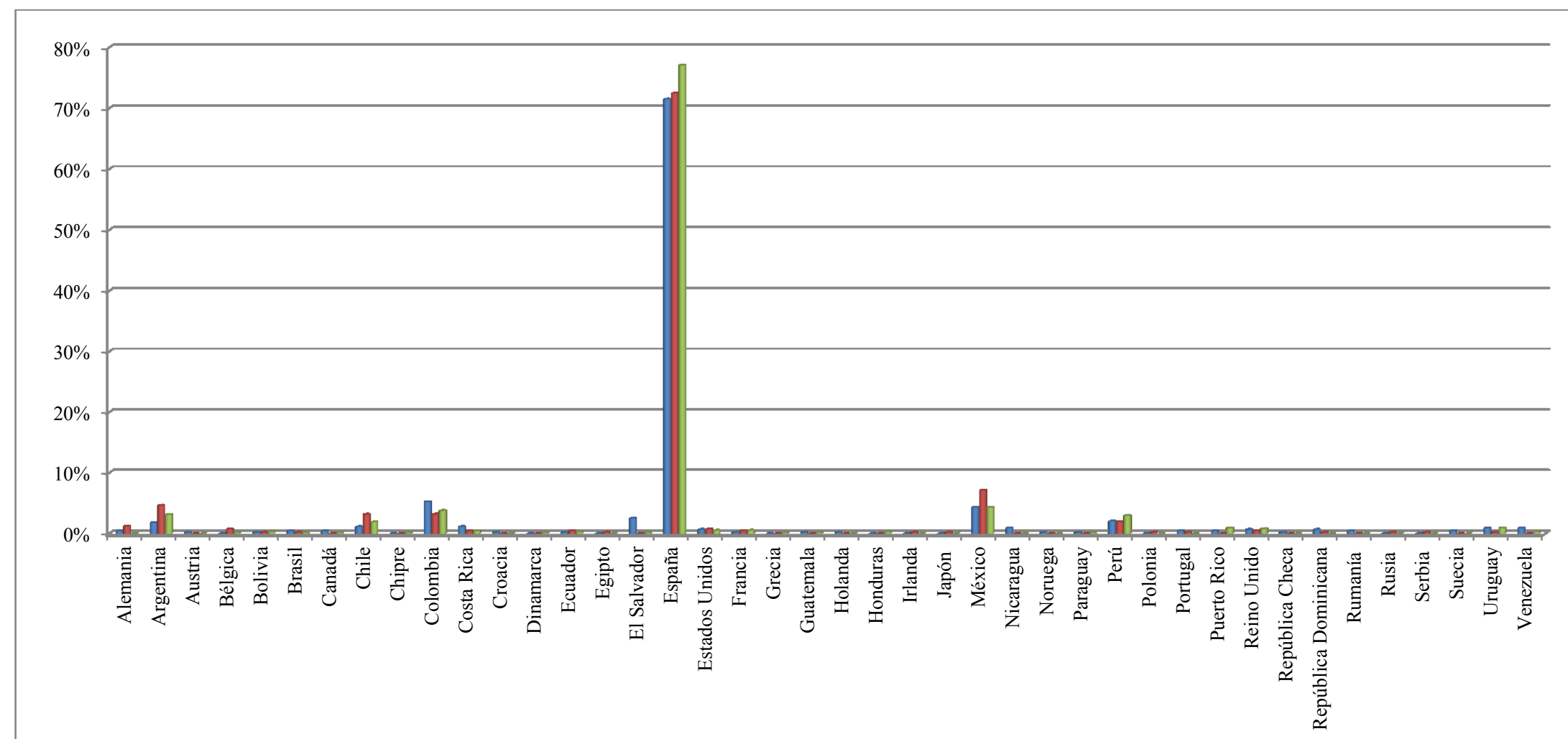

- Mujeres coraje. Desafíos en la Historia (curso 2013-2014) — Mujeres coraje. Maestras de la transgresión (curso 2014-2015) — Mujeres coraje. Subvirtiendo estereotipos (curso 2015-2016)

Fig. 2 Porcentaje de estudiantes por país de residencia

Fuente: Elaboración propia con los datos facilitados por el CENT (UJI)

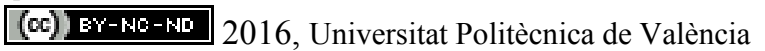
Congreso In-Red (2016) 

Ciertamente, el porcentaje más elevado reside en España, como puede observarse en la Fig. 3 , ya que la difusión realizada no posee tanto eco como otras plataformas más conocidas. Aun así podemos constatar como ya se ha comentado, a través de la Tabla 1, el gran número de países alcanzados. Consideramos que los datos demográficos son menos fiables, ya que el estudiantado no estaba obligado a contestar a este tipo de cuestiones.

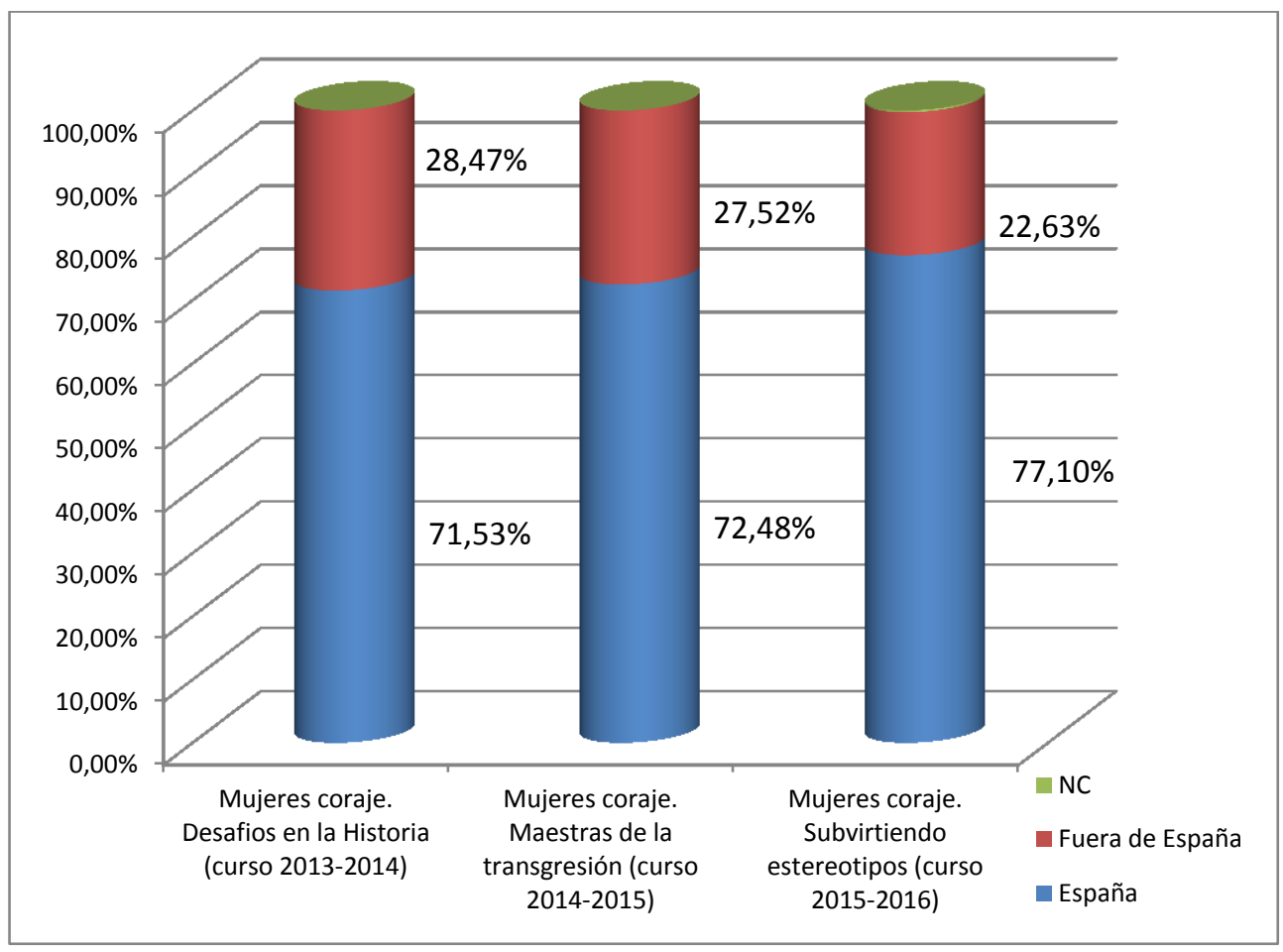

Fig. 3 Porcentaje de estudiantes residentes en España y en el extranjero

Fuente: Elaboración propia con los datos facilitados por el CENT (UJI)

Una vez analizados los países de origen, la lengua vehicular y el número de matriculación, el otro aspecto a tratar, más si hablamos de un MOOC específico en estudios de género, es el sexo de las personas matriculadas. En este punto, hemos detectado en todas las ediciones una brecha significativa entre mujeres y hombres matriculados. Si bien, en el primer «Mujeres coraje» la diferencia era importante, en las ediciones posteriores es todavía más relevante, como puede apreciarse en la Fig. 4. 


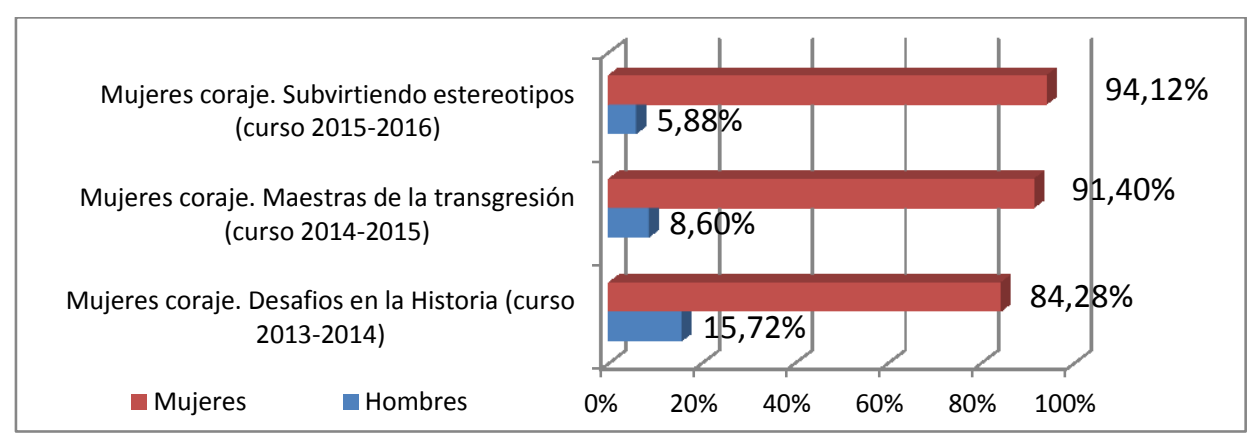

Fig. 4 Porcentaje por género del estudiantado inscrito en cada MOOC.

Fuente: Elaboración propia con los datos facilitados por el CENT (UJI).

\section{Resultados}

Teniendo en cuenta el análisis demográfico anterior debemos destacar aquello que caracteriza a este tipo de docencia, la diferencia plausible entre la inscripción, la participación efectiva-usuarios y usuarias que entraron en el MOOC durante el período de impartición- y la finalización.

Como puede observarse en la figura 5 la participación efectiva no ha variado de manera considerable de la primera edición a la última, realizada este año. Se ha mantenido más o menos estable, en un porcentaje satisfactorio y superior a lo esperado, teniendo en cuenta las características de este tipo de docencia. Así mismo, el estudiantado que finaliza el curso se encuentra en una horquilla que oscila del 14,81\% al 7,33\%, unas cifras que contrastan con la participación efectiva y la inscripción total como es habitual en este tipo de cursos. Aunque estos últimos números son llamativos entran dentro de los parámetros habituales, ya que no todo el estudiantado se inscribe pensando en finalizar realmente el curso. Debemos recordar que para muchos el objetivo es obtener materiales, recursos y un medio para el diálogo con otras personas interesadas en los mismos temas.

Por otro lado, cabe reseñar que en este último curso se han superado las cifras de la segunda edición. Estos datos fueron los que motivaron el cambio metodológico que, a nuestro entender, ha provocado una mayor participación.

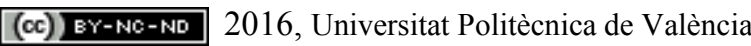




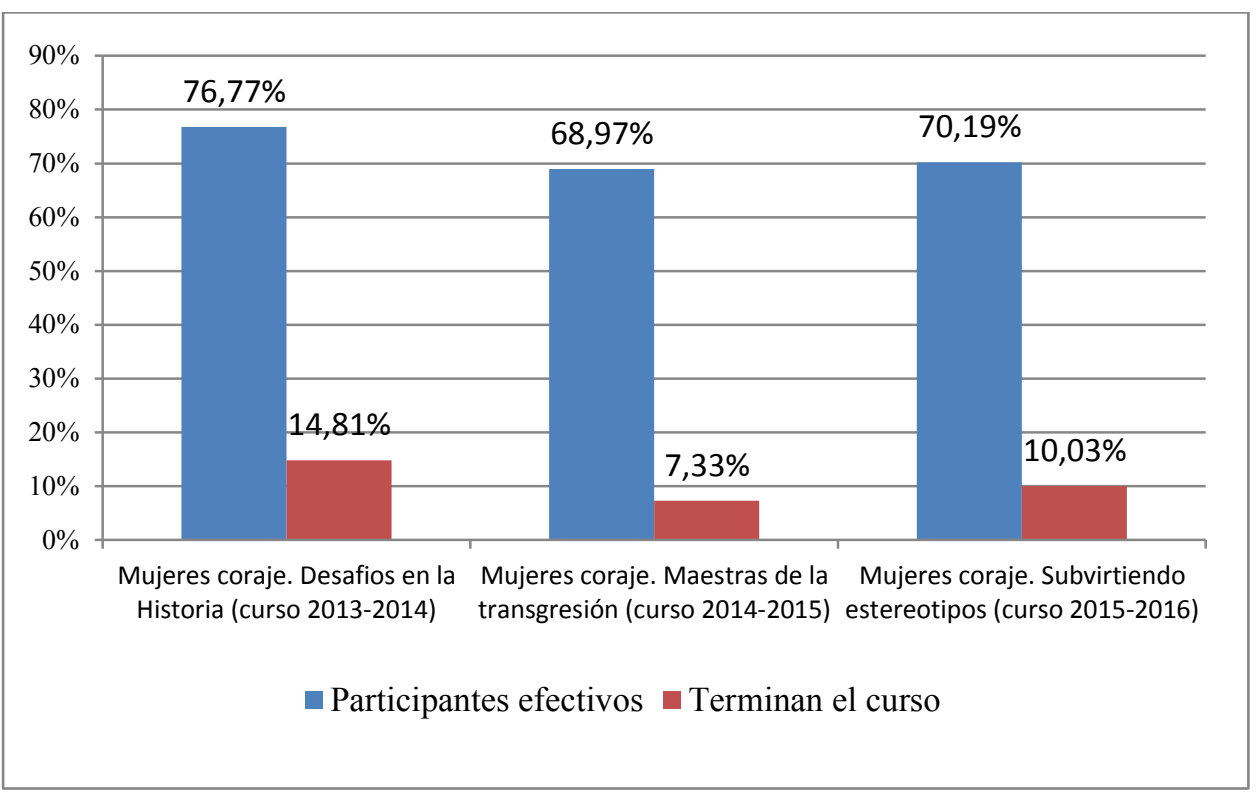

Fig. 5 Porcentaje de participación efectiva y de finalización del estudiantado en cada MOOC.

Fuente: Elaboración propia con los datos facilitados por el CENT (UJI).

\section{Conclusiones}

Nuestra experiencia nos ha demostrado lo importante que resulta evaluar las prácticas innovadoras en el ámbito educativo e introducir modificaciones tras dicho análisis. Esto se puede observar en el cambio experimentado en el último curso, acercándonos a los xMOOCs.

Los datos obtenidos están en la línea del resto de MOOCs en cuanto a la finalización del curso por parte del estudiantado. Son masivos, aunque Mujeres coraje -por lo ya expuesto- no llega a las cifras de las grandes plataformas, y por tanto, es imposible hacer un seguimiento de los participantes como si se tratara de una docencia online oficial.

En estos tres años hemos conseguido, en parte, alcanzar los objetivos marcados en su origen y que coinciden con los generales del conjunto universitario, dar visibilidad a los estudios de género.

Para finalizar, no debemos obviar que la mayoría de las y los inscritos se circunscriben al ámbito universitario, a pesar de ser masivos y gratuitos, sin la necesidad, en nuestro caso de conocimientos previos específicos. Esto indica la existencia de una brecha digital que parecía que este tipo de docencia iba a romper. En la actualidad, los MOOCs siguen siendo, para una gran mayoría, unos grandes desconocidos, puesto que se «encierran» en un ámbito muy concreto. Los datos obtenidos evidencian la falta de difusión por parte de las instituciones educativas implicadas y por tanto la falta de

\section{(cc) EY-NC-ND 2016, Universitat Politècnica de València}


comunicación con una sociedad que podría beneficiarse de estos recursos e instrumentos educativos.

\section{Referencias}

ADELL, Jordi (2014). «La insoportable levedad de los MOOC», 5 de diciembre. $<$ http://elbonia.cent.uji.es/jordi/2014/12/05/la-insoportable-levedad-de-los-mooc/> [Consulta: 25 de marzo de 2016].

ATENAS, Javiera (2015). «Modelo de democratización de los contenidos albergados en los MOOC». RUSC. Universities and Knowledge Society Journal, 12 (1). Págs. 3-14. <DOI: http://dx.doi.org/10-7238/rusc.v12i1.2031> [Consulta: 25 de marzo de 2016].

Despujol Zabala, Ignacio (2015). «Creación de MOOC. ¿Qué es un MOOC?». Youtube < https://www.youtube.com/watch?v=1ZFpBhXi6pc > [Consulta: 25 de marzo de 2016]

DownEs, Stephen (2012). «The Rise of MOOCs», 23 de abril. <http://www.downes.ca/post/57911> [Consulta: 25 de marzo de 2016].

Fernández Nadal, Carmen María y Caballero Guiral, Juncal (2015): «Mooc. Una nueva propuesta educativa en estudios de género» Beltrán ARANDES, Joaquín y Ripollés Meliá, María (ed.): V Jornada Nacional sobre estudios universitarios. I Taller de innovación educativa. Universitat Jaume I. Págs. 216-224.

GEA, Miguel (coord.) (2015). «Informe MOOC y criterios de calidad. Versión 1.0». En Jornadas CRUE TIC. <http://www.crue.org/TIC/Documents/InformeMOOC_CRUETIC_ver1\%200.pdf> [Consulta: 25 de marzo de 2016].

HERnÁNDEZ, Belén (2014). «A dos clics de la formación gratuita». El País, 3 de febrero de 2014.

Instituto Universitario de Estudios Feministas y de Género Purificación Escribano. UNIVERSITAT JAUME I DE CASTELLÓN. Mujeres coraje. $<$ http://mujerescoraje.uji.es/> [Consulta: 25 de marzo de 2016] [Institucional].

LANE, Lisa (2012). «Three Kinds of MOOCs», 15 de agosto. $<$ http://lisahistory.net/wordpress/2012/08/three-kinds-of-moocs $>$ [Consulta: 25 de marzo de 2016].

LuJÁn MORA, Sergio (2012). «Breve (muy breve) historia de los MOOCs». Youtube < https://www.youtube.com/watch?v=sZCyBVEw5Xw> [Consulta: 25 de marzo de 2016].

PÉREZ DE PABLOS, Susana (2014). «La tecnología permitirá que los universitarios construyan sus títulos con partes de asignaturas». El País, 30 de diciembre de 2014.

Pernías Peco, Pedro y Luján Mora, Sergio (s.f.). «Los MOOC: orígenes, historia y tipos». Comunicación y Pedagogía. Especial MOOC. $\mathrm{N}^{\circ}$ 269-270. Págs. 41-47. $<$ http://www.centrocp.com/los-mooc-origenes-historia-y-tipos/> [Consulta: 25 de marzo de 2016]

SCOPEO (2013). «SCOPEO INFORME No 2 : MOOC:Estado de la situación actual, posibilidades, retos y futuro». Junio 2013. Scopeo Informe $\mathrm{N}^{\mathrm{o}} 2 . \quad<\mathrm{http} / / / \mathrm{scopeo}$. usal.es/wpcontent/uploads/2013/06/scopeoi002.pdf> [Consulta: 25 de marzo de 2016].

SiLIÓ, Elisa (2014). «El conocimiento en un vídeo de dos minutos». El País, 27 de noviembre de 2014.

(cc) EY-NC-ND 2016, Universitat Politècnica de València 
UNIVERSIA (2015). «Ventajas del aprendizaje online», 20 de abril $<$ http://noticias.universia.es/educacion/noticia/2015/04/20/1123518/ventajas-aprendizajeonline.html> [Consulta: 25 de marzo de 2016].

UNIVERSIDAD AUTÓNOMA DE BARCELONA. Representaciones culturales de las sexualidades. $<$ https://www.coursera.org/learn/representaciones-culturales> [Consulta: 25 de marzo de 2016] [Institucional].

UNIVERSIDAD DE CHILE. Nuevas miradas sobre género $y$ etnicidad III. $<$ http://uabierta.uchile.cl/courses/Universidad_de_Chile/UCh-5/2016/about $>$ [Consulta: 25 de marzo de 2016] [Institucional].

Universitat JAUme I DE CAStellón. Mujeres coraje. Subvirtiendo estereotipos. $<$ http://mooc.uji.es/course/mujeres-coraje-subvirtiendo-estereotipos/\#.Vv5DbPmLTIU > [Consulta : 25 de marzo de 2016] [Institucional].

UniversitAT JAUME I DE CASTELlÓN. Mujeres coraje. Maestras de la transgresión. $<$ http://ocw.uji.es/curso/910874> [Consulta: 25 de marzo de 2016] [Institucional].

Universitat Jaume I DE CAStellón. Mujeres coraje. Desafios en la historia. $<$ http://ocw.uji.es/curso/680551> [Consulta: 25 de marzo de 2016] [Institucional].

VÁZQUEZ-CANO, Esteban (2013). «El videoartículo: nuevo formato de divulgación en revistas científicas y su integración en MOOCs». Comunicar, No 41, V. XXI. Págs. 83-91. <DOI: http://dx.doi.org/10.3916/C41-2013-08> [Consulta: 25 de marzo de 2016].

(cc) EY-NC-ND 2016, Universitat Politècnica de València

Congreso In-Red (2016) 